\title{
In-situ TEM observation of the growth process of carbon nanomaterials by laser irradiation
}

Ryosuke Senga $^{1}$, Yung-Chang Lin ${ }^{1}$, Sapna Sinha ${ }^{1}$, Takeshi Kaneko ${ }^{2}$, Norihiro Okoshi ${ }^{2}$, Takeo Sasaki ${ }^{2}$, Shigeyuki Morishita ${ }^{3}$, Hidetaka Sawada ${ }^{4}$, Sang Tae Park ${ }^{5}$ and Kazu Suenaga ${ }^{6}$

${ }^{1}$ AIST, United States, ${ }^{2}$ JEOL Ltd., United States, ${ }^{3}$ JEOL Ltd., Tokyo, Japan, ${ }^{4}$ JEOL Ltd., Akishima, Japan, ${ }^{5}$ IDES, Inc., United States, ${ }^{6}$ Osaka University, United States

Laser ablation, arc discharge, and chemical vapor deposition methods have been used to synthesize carbon nanomaterials, and it is now possible to control their structure, such as the diameter and number of layers of carbon nanotubes, with high precision. The growth mechanism of carbon nanomaterials has been discussed both experimentally and theoretically for many years, and various models have been proposed [1]. In terms of experiments using transmission electron microscope (TEM), ex-situ observations, where growth is stopped and the catalyst is evaluated during the reaction, have identified the nucleation points of carbon nanotubes [2]. Furthermore, in-situ observations under a gas atmosphere using an environmental TEM have successfully determined the composition and crystal structure of the catalyst during the reaction [3-5].

However, it is still unclear what happens to the catalyst at the moment of nucleation and how the chirality and the number of layers are determined, because there are few examples of direct observation of carbon precipitation on the catalyst. In addition, the behavior of the catalyst under laser irradiation has not yet been clarified because of the lack of direct observation of the growth process in the laser ablation method.

In this study, we have succeeded in in-situ observation of the formation of tube- or onion-shaped carbon nanostructures with metal catalysts using the laser irradiation technique in TEM (Luminary Micro). This technique, developed by JEOL Ltd. and IDES Inc., enables us to irradiate a laser beam nearly parallel to the electron beam onto a sample while simultaneously observing it with an electron beam (Fig. 1). By controlling the laser power and minimizing the vibration of the sample, in-situ observation under the laser beam can be performed with high spatial resolution. In this experiment, a JEOL JEM-ARM300F was used with an acceleration voltage of $80 \mathrm{kV}$. All TEM images and movies were acquired using Gatan Oneview camera.

We first prepared a thin carbon film on which ferrocene molecules were deposited by a vacuum heating process. When the sample was irradiated with a laser at a wavelength of $577 \mathrm{~nm}$ and an output power of $50 \mathrm{~mW}$, the ferrocene decomposed instantly to form iron nanoparticles. Then, the iron nanoparticles randomly moved on the carbon film while changing their shape, forming tube-like structures along their path under the pulse laser $(1 \mathrm{kHz} \sim 5 \mathrm{kHz})$. The surface of the iron particles repeatedly changes between two states: spherical and faceted (Fig. 2). Interestingly, the graphite structures are more likely to form at the faceting moment, suggesting a correlation between the chirality of the nanotubes and the orientation of the facets.

This method is the only one that allows us to directly observe various physical phenomena and chemical reactions induced by lasers/lights and has the potential to bring paradigm shifts in a wide range of fields, including photochemistry and biology, as well as materials science. 


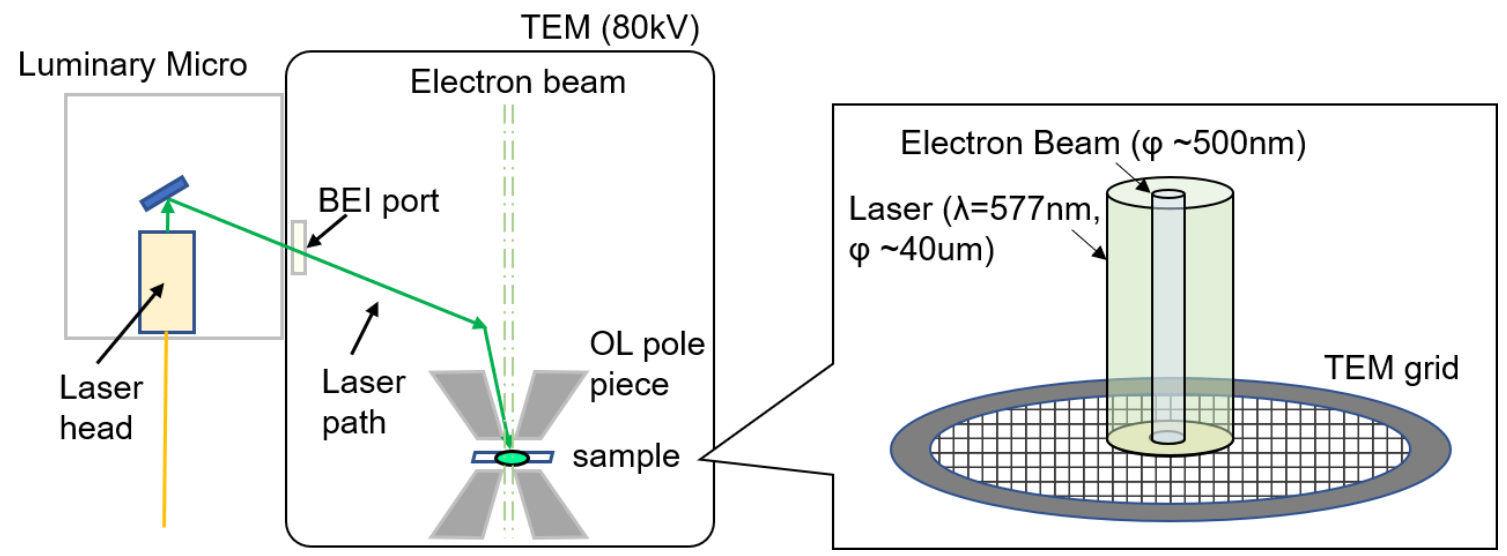

Figure 1. Schematic of the laser irradiation system in TEM (Luminary Micro). The center of the electron beam and the laser are adjusted to be the same.


Figure 2. Behavior of iron nanoparticles under laser irradiation (The energy density is approximately 4 $\mathrm{mJ} / \mathrm{cm} 2$ ). The nanoparticles move from the top to the bottom of the panels while changing their shape. The graphitized structures are formed along the travel path.

\section{References}

[1] Das et al. Nanoscale Research Letters 11, (2016) 510

[2] H. Zhu et al., Small 1, 1180 (2005)

[3] H. Yoshida et al., Nano Letters 8, 2082 (2008)

[4] S. Hofmann et al., Nano Letters 7, 602 (2007)

[5] Y. Wang et al., ACS Nano 14, 16823 (2020) 